\title{
Evaluating the Effect of Mobility Speed on the Performance of three Handover Algorithms in Long Term Evolution Networks
}

\section{*OSIFEKO, MO; OKUBANJO, AA; ABOLADE, OR; OYETOLA, OK; OYEDEJI, AO; SANUSI, OI}

\author{
Department of Computer and Electrical Engineering, Olabisi Onabanjo University, Nigeria. \\ *Corresponding Author: osifeko.martins@oouagoiwoye.edu.ng
}

\begin{abstract}
This work uses NS3 simulation to study the effect of mobility speed on the performance of three handover algorithms in Long Term Evolution (LTE) Networks. A realistic multi-cell LTE network was set up using NS3 simulation software. Mobility models were used to vary the location of the User Equipment (UE), hence triggering handover events across the network. The performance was measured using Signal Interference Noise Ratio (SINR) and number of completed handovers. Result revealed that at a speed between the ranges of $0-3 \mathrm{~km} / \mathrm{h}$, the Integrative algorithm performed best while at $4-60 \mathrm{~km} / \mathrm{h}$, the performance of the A3RSRP algorithm was the best with an average value of $95 \mathrm{~dB}$. Also, at an increased speed within the range of $60-120 \mathrm{~km} / \mathrm{h}$, the Integrative algorithm had a slightly better performance than the A3RSRP. However, at a speed above $120 \mathrm{~km} / \mathrm{h}$, the integrative algorithm performed best with an SINR of $120 \mathrm{~dB}$. In terms of completed handovers, the Integrative algorithm had the least number of completed handovers throughout the entire range of considered speeds. Thus, we establish that mobility speed has a significant effect on the performance of handover algorithms.
\end{abstract}

DOI: https://dx.doi.org/10.4314/jasem.v22i4.11

Copyright: Copyright $(2018$ Osifeko et al. This is an open access article distributed under the Creative Commons Attribution License (CCL), which permits unrestricted use, distribution, and reproduction in any medium, provided the original work is properly cited.

Dates: Received: 10 March 2018; Revised: 15 April: 2018; Accepted: 20 April 2018

Keywords: LTE handover, LTE UE Speed, Integrative Algorithm, Power Budget Algorithm

The Long-Term Evolution (LTE) system was designed with the aim of providing a higher data rates and lower latency under various mobility conditions (Dimou et al., 2009). According to (3GPP TR 25.913), the LTE system is expected to provide mobility support for User Equipment (UE) up to speeds of $500 \mathrm{~km} / \mathrm{h}$ while maintaining an uninterrupted provision of high data rates and services. Mobility at high speed has always been a challenge in wireless networks and LTE was designed to overcome this challenge. To accomplish this purpose, LTE must minimize delay and packet loss in voice transmission and ensure reliability in data transmission during high-speed scenarios. In lieu of this, optimizing the handover procedure to get the required performance is considered as one important issue in mobile networks (Hämäläinen, 2011).

LTE Handover is a process that transfers a UE from one evolved NodeB (eNodeB) to another eNodeB or one sector to another sector within the same eNodeB due to perceived better cell coverage from the target eNodeB (Lin et al, 2011a). This goal is achieved by analyzing a periodic or event triggered downlink received signal strength (RSS) and carrier-tointerference ratio (CIR) measurements from the UEs. The eNodeB then decides based on the received parameters on whether to handover the UE to the neighboring eNodeB or keep the UE connected to it. The decision-making process is controlled by an efficient handover algorithm as it enhances the system capacity and the service quality cost effectiveness. The performance of the LTE handover scheme depends majorly on the handover algorithm in use (Hans et al, 2014). Due to this fact, researchers have channeled efforts at optimizing existing algorithms while some new ones have been developed. Three of the numerous algorithms that have become popular in LTE networks include (i) Power Budget Handover Algorithm (Anas et al, 2007) (ii) RSS-based handover with time-totrigger (TTT) window (Anas et al, 2007) (iii) Integrator Handover Algorithm (Zheng and Wigard, 2008).

Over the last decade, researchers have carried out works aimed at evaluating the performances of the various handover algorithms. However, from a survey of the literature, there is a scarcity of work regarding the effect of UE speeds on the performance of the various handover algorithms. An optimized value for each handover algorithms was determined under different user speeds in Lin et al, (2011). The result of the work showed that an optimized handover algorithm can reduce an unnecessary number of handovers with maximized system throughput. The work further recommended that the optimized version of the algorithms be used in the further study of performance evaluation of handover algorithms in LTE system. A Coordinated multipoint (CoMP)-based Handover Algorithm was proposed and compared with the LTE Hard Handover Algorithm in Wang and Tung (2016) while a new handover optimization algorithm for LTE network based on fuzzy logic was presented 
in Saeed et al, (2016). The proposed handover optimization technique was evaluated and compared with four other handover algorithms. The proposed algorithm performed better than the other algorithms by achieving a minimum number of handover and having a maximum throughput than the selfoptimization technique. An efficient handover mechanism based on fuzzy logic was proposed in Saeed et al, (2017). It was evaluated in terms of handovers completed, system throughput, and system delay based on UE speed. The suggested technique results were compared with other well-known handover algorithms at different UE speed scenarios. Results showed that the suggested technique effectively improved network performance by minimizing the average completed handovers while also providing an increased throughput. From the literature reviewed, it can be observed that most of the works did not consider the effect of the various handover algorithms on the downlink and uplink SINR of the UEs. This work aims to compare the performance of three well-known algorithms that includes the Integrative, Received Signal Strengthbased TTT Window, and Power Budget Handover Algorithm using downlink SINR, uplink SINR and handover completed as performance metrics.

\section{MATERIALS AND METHODS}

Power Budget Handover Algorithm: The Power Budget Handover Algorithm is an effective algorithm that uses two variables to make handover decisions handover margin (HOM) and Time to Trigger (TTT) timer (Lin et al, 2011b). A handover margin is a variable that represents the threshold for the difference in received signal strength between the serving and the target cell. Before the eNodeB considers the UE for a handover, the perceived signal strength of the target cell $\mathrm{RSRP}_{\mathrm{T}}$ must be greater than the signal strength of the serving cell $\mathrm{RSRP}_{\mathrm{s}}$ plus the handover margin provided the TTT condition has been satisfied. The TTT is used to avoid a ping-pong condition by restricting the handover action from being triggered within a certain time duration. A handover action can only be performed after TTT is satisfied.

$\mathrm{RSRP}_{\mathrm{T}}>\mathrm{RSRP}_{\mathrm{S}}+\mathrm{HOM}$

Where $\mathrm{RSRP}_{\mathrm{T}}$ and $\mathrm{RSRP}_{\mathrm{S}}$ are received signal strength from the target and serving cell, respectively and $\mathrm{HOM}$ is the handover margin.

Received Signal Strength-Based TTT Window: The UE measures the RSS that comprises of the path loss, antenna gain, log-normal shadowing, and fast fading averaged over all the reference symbols within measurement bandwidth $\mathrm{BW}_{\mathrm{m}}$. The filtered RSS is then measured periodically at the time (Tm) as the output of a first order infinite impulse response (IIR) filter as defined in (3). The relative influence on RSS of the recent measurement and older measurements is controlled by the forgetting factor (Anas, et al, 2007)
$\beta=\frac{\mathrm{T}_{\mathrm{m}}}{\mathrm{T}_{\mathrm{u}}}$

Where, $T_{u}$ is an integer multiple of $T_{m}$ and $T_{m}$ is the handover measurement period.

$\operatorname{RSS}(\mathrm{nTm})=\beta * \operatorname{RSS}(\mathrm{nTm})+(1-\beta) \operatorname{RSS}\left((\mathrm{n}-1) \mathrm{T}_{\mathrm{m}}\right)$

The handover decision is based on the RSS and is executed if the condition in (4) is satisfied

$\operatorname{RSS}\left(\mathrm{nT}_{\mathrm{u}}\right) \cdot \mathrm{TS} \geq \mathrm{RSS}\left(\mathrm{nT}_{\mathrm{u}}\right) \cdot \mathrm{sS}+\mathrm{HOM}$

Where HOM is a constant threshold value, $\left(\mathrm{nT}_{\mathrm{u}}\right)_{\cdot \mathrm{TS}}$ and $\left(\mathrm{nT}_{\mathrm{u}}\right)_{\mathrm{SS}}$ are the filtered RSS of the target sector (TS) and the filtered RSS of the serving sector (SS) at $\mathrm{n}^{\text {th }} \mathrm{Tu}$ interval, respectively.

Integrator Handover Algorithm: This algorithm works by integrating the RSRP differences of the source and target cell by using an infinite impulse response (IIR) filter. The handover decision is then made according to the triggering condition between the filtered RSRP differences and the triggering threshold. There are 3 parts in this algorithm, RSRP difference calculation, filtered RSRP difference computation, and handover decision (Zheng and Wigard, 2008; Lin et al, 2011a). The RSRP difference calculation is presented in equation (5)

$\operatorname{DIFs}_{\_}(\mathrm{t})=\operatorname{RSRP}_{\mathrm{T}}(\mathrm{t})-\operatorname{RSRP}_{\mathrm{s}}(\mathrm{t})$

Where, $\operatorname{RSRP}_{\mathrm{T}}(\mathrm{t})$ and $\operatorname{RSRP}_{\mathrm{S}}(\mathrm{t})$ are received signal strength from the target and serving cell at time $t$ respectively.

DIFs $\mathrm{j}(\mathrm{t})$ is the RSRP difference of the user $j$ at serving cell $s$ at time $t$. The filtered RSRP difference computation is expressed as

FDIFs $\mathrm{j}(\mathrm{t})=(1-\alpha)$ FDIFs $\mathrm{j}(\mathrm{t}-1)+\alpha \operatorname{DIFs}_{-} \mathrm{j}(\mathrm{t})$

Where $\alpha$ is a proposed variable with constraint $0 \leq \alpha$ $\leq 1$ and FDIFs $\mathrm{j}(\mathrm{t})$ is the filtered RSRP difference value of user $j$ at serving cell $s$ at time $t$, and DIFs $\mathrm{j}(\mathrm{t})$ is the RSRP difference value calculated in equation 5 .

Methods: To evaluate the effect of speed on the performance of the three (3) handover algorithms, the NS-3 simulator is used. A network comprising of nine (9) eNodeBs and ten (10) UE was setup using the network simulator. The UEs were then made to roam the network at 5 randomly selected speeds of $3 \mathrm{~km} / \mathrm{h}$, $30 \mathrm{~km} / \mathrm{h}, 60 \mathrm{~km} / \mathrm{h}, 120 \mathrm{~km} / \mathrm{h}$ and $170 \mathrm{~km} / \mathrm{h}$. At each of the speed, the three algorithms were then made to control the handover procedure while the corresponding key performance indicators (KPIs) that determine the performance of the handover algorithms were measured. 


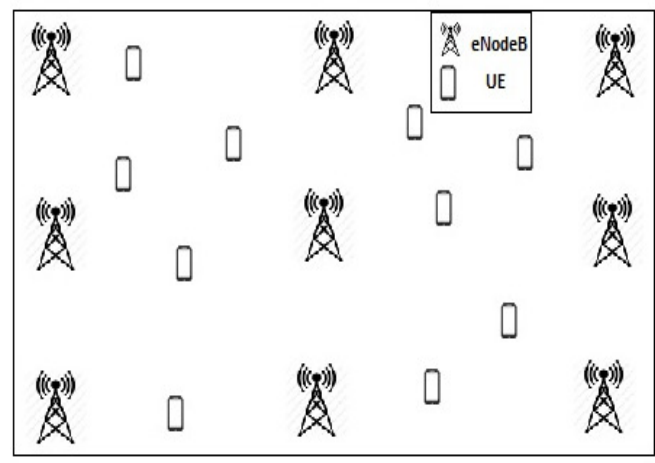

Fig 1: Topology of the Simulated Network

The two KPIs are Number of completed handovers and Signal to Interference plus Noise Ratio (SINR). The channel model includes path loss, log-normal shadowing, and frequency selective fast fading. The log-normal shadowing samples are spatially correlated using a negative exponential function.

9 sites of tri-sectored macro eNodeBs (i.e. 27 macrocells) deployed in hexagonal layout with $500 \mathrm{~m}$ intersite distance. UEs are randomly distributed around the sites and attach to the network automatically using idle mode cell selection. The duration of the simulation is 30 seconds to allow the UEs travel far enough to trigger some handovers. Other simulation parameters are as shown in Table 1.

The eNodeBs and UEs were configured with a transmit power $\mathrm{Tx}$ of $46 \mathrm{dBm}$ and $10 \mathrm{dBm}$ respectively. Evolved Packet Core (EPC) mode will be used because the $\mathrm{X} 2$ handover procedure requires it to be enabled. Full-buffer downlink and uplink traffic were used in the simulation both in $5 \mathrm{MHz}$ bandwidth, using TCP protocol and Proportional Fair scheduler.

Table 1: Simulation Parameters

\begin{tabular}{|c|c|c|}
\hline $\begin{array}{l}\text { Parameter } \\
\text { Simvlation Time }\end{array}$ & $\begin{array}{l}\text { Assumptions } \\
30 \text { seconds }\end{array}$ & $\begin{array}{c}\text { Description } \\
30 \text { seconds simvlation time }\end{array}$ \\
\hline nMacroEnbSites & 9 & Number of eNodeBs \\
\hline inter $\delta$ iteDis tance & $500 \mathrm{~m}$ & Distance between adjacent eNodeBs \\
\hline eNodeB TxPower & $46 \mathrm{dBm}$ & Transmit power for the eNodeB \\
\hline $\mathrm{nUES}$ & 10 & Number of UEs \\
\hline $\begin{array}{l}\text { UeTxPower } \\
\text { Speed of UE }\end{array}$ & $\begin{array}{l}10 \mathrm{dBm} \\
3,30,60,120 \& 170 \mathrm{~km} / \mathrm{h}\end{array}$ & $\begin{array}{l}\text { Transmit power for the UE } \\
\text { Roaming speed of the UE }\end{array}$ \\
\hline $\begin{array}{l}\text { ns } 3:: \text { LteHelper: Handove } \\
\text { rAlgorithm } \\
\text { ns } 3:: \text { LteHelper: Schedule }\end{array}$ & 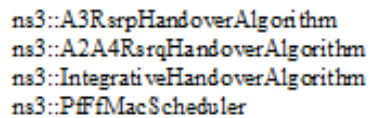 & $\begin{array}{l}\text { The handover algorithms that were used } \\
\text { in the simvlation. } \\
\text { Proportional Fair scheduler }\end{array}$ \\
\hline
\end{tabular}

\section{RESULTS AND DISCUSSION}

Signal Interference Noise Ratio (SINR) is a measure of signal quality defined by the UE vendor however, it is not reported to the network. SINR is used by operators and the LTE industry in general, as it better quantifies the relationship between RF conditions and Throughput. UEs typically use SINR to calculate the Channel Quality Indicator (CQI) they report to the network.

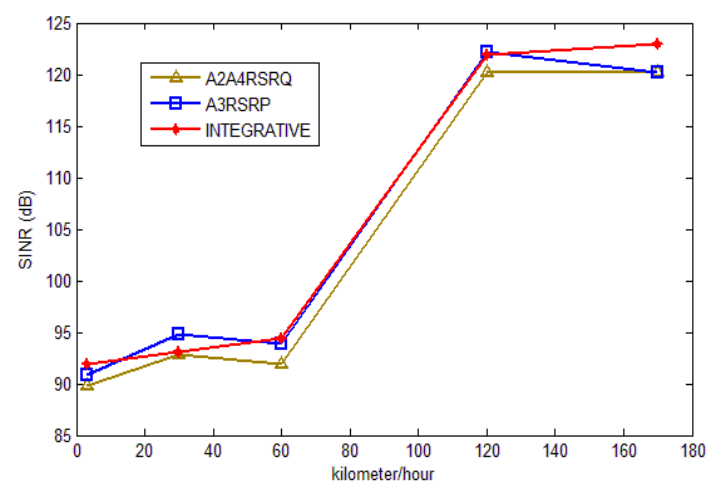

Fig 2: Downlink SINR Measurement for the three algorithms at various speeds

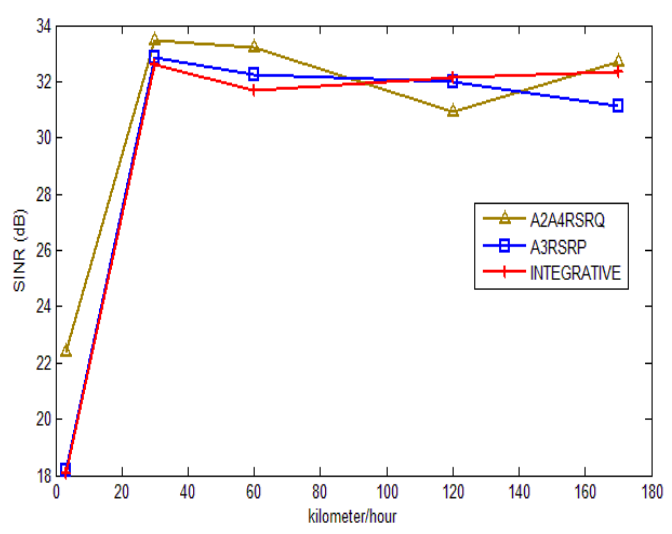

Fig 3: Uplink SINR Measurement for the three algorithms at various speeds

In terms of the downlink SINR, result from Figure 2 revealed that at a speed between the range of $0-3 \mathrm{~km} / \mathrm{h}$, the Integrative algorithm performed best while at 4 $60 \mathrm{~km} / \mathrm{h}$, the performance of the A3RSRP algorithm was the best with an average value of $95 \mathrm{~dB}$. Also, at an increased speed within the range of $60-120 \mathrm{~km} / \mathrm{h}$, the Integrative algorithm had a slightly better performance than the A3RSRP. However, at a speed above $120 \mathrm{~km} / \mathrm{h}$, the integrative algorithm performed best with an SINR of $120 \mathrm{~dB}$. A peak SINR value of 
$123 \mathrm{~dB}$ was observed for the Integrative algorithm at a speed above $140 \mathrm{~km} / \mathrm{h}$.

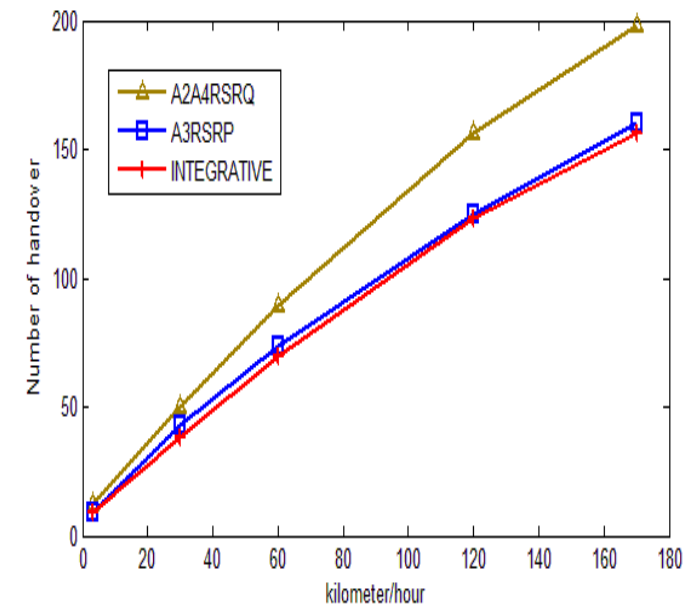

Fig 4: Number of completed handovers for the three algorithms at various speeds

In terms of the uplink SINR, result from Figure 3 revealed that at a speed between the range of $0-60$ $\mathrm{km} / \mathrm{h}$, the performance of the A2RSRQ algorithm was the best with a peak value of $33 \mathrm{~dB}$. However at a speed between $90-140 \mathrm{~km} / \mathrm{h}$, the performance of the algorithm dropped below the other two algorithms. A steady rise in the SINR could also be observed at a speed between 0 and $30 \mathrm{~km} / \mathrm{h}$.

The result further revealed that at a speed above $30 \mathrm{~km} / \mathrm{h}$ the SINR only varied between $30 \mathrm{~dB}$ and 33 $\mathrm{dB}$. Regarding the number of completed handovers, the Integrative algorithm had the least number of completed handovers throughout the entire range of considered speeds.

According to 3GPP, this indicates a better performance as a handover algorithm is expected to minimize the number of handovers taking place in a network due to its signaling overhead and associated effect on network resources. It is worthy of note that the A3RSRP algorithm also had a good performance and was closely tied to the Integrative algorithm throughout the range of speeds considered in this work.

Conclusion: In this work, a study of the effect of mobility speed on the performance of handover algorithms in LTE Networks was carried out using the NS3 simulator. Results established that speed of mobility has a significant effect on the performance of handover algorithms. The result of this research work can be used by researchers when developing new handover algorithms by ensuring their algorithms operates efficiently at various mobility speeds.

\section{REFERENCES}

3GPP TR 25.913, "Requirements for evolved UTRA (E-UTRA) and evolved UTRAN (E-UTRAN)," version 7.3.0.

Anas, M; Calabrese, FD; Mogensen, PE; Rosa, C; Pedersen, KI, (2007). Performance evaluation of received signal strength based hard handover for UTRAN LTE. In Vehicular Technology Conference. VTC2007-Spring. IEEE 65th:10461050.

Dimou, K; Wang, M; Yang, Y; Kazmi, M; Larmo, A; Pettersson, J; Muller, W; Timner, Y,(2009). Handover within 3GPP LTE: design principles and performance. In Vehicular Technology Conference Fall (VTC 2009-Fall). IEEE 70th:1-5.

Hämäläinen J, (2011). Optimizing handover performance in LTE networks containing relays (Doctoral dissertation, Aalto University).

Hans, A; Sharma, A; Kumar, K; Singh, N (2014). An overview of handoff procedure in LTE technology. In Medical Imaging, m-Health and Emerging Communication Systems (MedCom). IEEE International Conference: 391-394.

Lin, CC; Sandrasegaran, K; Ramli, HAM; Basukala, R; Patachaianand, R; Chen, L; Afrin, TS, (2011a). Optimization of handover algorithms in 3GPP long term evolution system. In Modeling, Simulation and Applied Optimization (ICMSAO). 4th International Conference: 1-5.

Lin, CC; Sandrasegaran, K; Ramli, HAM; Basukala. R; (2011b). Optimized performance evaluation of LTE hard handover algorithm with average RSRP constraint. arXiv preprint arXiv:1105.0234. https://doi.org/10.5121/ijwmn.2011.3201

Saeed, M; Kamal, H; El-Ghoneimy, M (2016). A new fuzzy logic technique for handover parameters optimization in LTE. In Microelectronics (ICM), 28th IEEE International Conference: 53-56.

Saeed, M; El-Ghoneimy, M; Kamal H. (2017). An enhanced fuzzy logic optimization technique based on user mobility for LTE handover. In 34th National IEEE Radio Science Conference (NRSC):230-237.

Wang YH; Tung YC. (2016). A CoMP-based Handover Algorithm in LTE-Advanced. Journal of Computers. 27(4):149-160.

Zheng N; Wigard J (2008). On the performance of integrator handover algorithm in LTE networks. In Vehicular Technology Conference, 2008. VTC 2008-Fall. IEEE 68th:1-5. 